\title{
Detection of SARS-CoV-2 in the ocular surface in different phases of COVID-19 patients in Shanghai, China
}

\author{
Meiyan $\mathrm{Li}^{1 \#}$, Yaling Yang ${ }^{2 \#}$, Taiwen $\mathrm{He}^{2 \#}$, Ruoyan Wei ${ }^{1}$, Yinzhong Shen ${ }^{3}$, Tangkai Qi ${ }^{3}$, Tian Han $^{1}$, \\ Zhigang Song ${ }^{4}$, Zhaoqin Zhu ${ }^{5}$, Xiaopeng $\mathrm{Ma}^{6}$, Jing Zhao ${ }^{1}$, Yixiao Lin ${ }^{3}$, Yang Shen ${ }^{1}$, Weiming Yang ${ }^{7}$, \\ Keqing Zhao ${ }^{8}$, Hongzhou $\mathrm{Lu}^{3}$, Xingtao Zhou ${ }^{1 \wedge}$
}

${ }^{1}$ Department of Ophthalmology and Optometry, Eye and ENT Hospital, Fudan University, Shanghai, China; NHC Key Laboratory of Myopia (Fudan University), Shanghai, China; Shanghai Research Center of Ophthalmology and Optometry, Shanghai, China; ${ }^{2}$ Department of Ophthalmology, Shanghai Public Health Clinical Center, Shanghai, China; ${ }^{3}$ Department of Infectious Diseases and Immunology, Shanghai Public Health Clinical Center, Shanghai, China; ${ }^{4}$ Department of Pathogen Diagnosis and Biosafety, Shanghai Public Health Clinical Center, Shanghai, China; ${ }^{5}$ Department of Laboratory Medicine, Shanghai Public Health Clinical Center, Shanghai, China; ${ }^{6}$ Shanghai Research Institute of Acupuncture and Meridian, Shanghai University of Traditional Chinese Medicine, Shanghai, China; ${ }^{7}$ Department of Ophthalmology, Children's Hospital of Fudan University, Shanghai, China; ${ }^{8}$ Department of Otolaryngology, Eye and ENT Hospital, Fudan University, Shanghai, China

Contributions: (I) Conception and design: X Zhou, H Lu; (II) Administrative support: X Zhou, H Lu; (III) Provision of study materials or patients: Y Yang, T He, H Lu; (IV) Collection and assembly of data: All authors; (V) Data analysis and interpretation: M Li, R Wei, Y Yang, T He, Y Shen, T Qi, T Han, X Ma, J Zhao, Y Lin, Y Shen; (VI) Manuscript writing: All authors; (VII) Final approval of manuscript: All authors.

\#These authors contributed equally to this work as co-first authors.

Correspondence to: Hongzhou Lu, MD, PhD. Department of Infectious Diseases and Immunology, Shanghai Public Health Clinical Center, 2901 Caolang Road, Shanghai 201508, China. Email: luhongzhou@fudan.edu.cn; Xingtao Zhou, MD, PhD. Department of Ophthalmology and Optometry, Eye and ENT Hospital of Fudan University, Myopia Key Laboratory of the Health Ministry, No. 83 Fenyang Road, Shanghai 200031, China. Email: doctzhouxingtao@163.com.

Background: To investigate the temporal pattern of severe acute respiratory syndrome coronavirus 2 (SARS-CoV-2) presence on ocular surfaces using conjunctival swabs in coronavirus disease 2019 (COVID-19) patients.

Methods: This study included 59 patients (32 newly admitted and 27 hospitalized for $\geq 2$ weeks) with a COVID-19-confirmed diagnosis at the Shanghai Public Health Clinical Center from March 3, 2020, to March 21, 2020. Conjunctival swab samples were collected from both eyes of all the 59 patients and were tested by reverse transcription polymerase chain reaction (RT-PCR) assay. The range of sampling time lies widely between 1 and 50 days since symptom onset.

Results: Among the 32 newly admitted patients, positive RT-PCR results for SARS-CoV-2 in conjunctival swab samples were reported in 2 patients (one eye for each) without ocular discomfort, but 1 positive case had conjunctival congestion. The positive results were detected on Day 5 for 1 patient and Day 7 for the other, but repeated tests after 1 week were negative for both patients. All 27 patients who had been hospitalized for $\geq 2$ weeks had negative test results. The mean time from symptom onset to sampling of 2 positive cases was significantly less than that of 57 negative cases $(\mathrm{P}<0.001)$.

Conclusions: SARS-CoV-2 on the ocular surface can be detected in the early phase of COVID-19. The risk of ocular transmission remains and might be higher in the early phase.

Keywords: Coronavirus disease 2019 (COVID-19); infection; ocular surface; severe acute respiratory syndrome coronavirus 2 (SARS-CoV-2); transmission

^ ORCID: 0000-0002-3465-1579. 
Submitted Aug 21, 2020. Accepted for publication Oct 30, 2020.

doi: $10.21037 /$ atm-20-6026

View this article at: http://dx.doi.org/10.21037/atm-20-6026

\section{Introduction}

An outbreak of a novel coronavirus, severe acute respiratory syndrome coronavirus 2 (SARS-CoV-2), began in December 2019 and has since spread around the world as a global pandemic. It has posed significant threats to international health. During the outbreak, healthcare workers are reported among the highest risk groups (1). It was first reported by the Chinese media that Dr. Guangfa Wang, a national expert on the panel for the early investigations of the disease in Wuhan, China, had a red eye being the initial symptom before the onset of pneumonia. Notably, there are anecdotal reports of three ophthalmologists in China being infected with SARS-CoV-2 when treating coronavirus disease 2019 (COVID-19) patients with eye diseases, who subsequently died from the disease (2). For those health professionals in direct or indirect contact with ocular surfaces of patients, such as ophthalmologists, it is clinically important to know whether they are exposed to the risk of SARS-CoV-2 infection via an ocular route.

To date, previous studies have indicated that $0.8 \%$ of patients infected with COVID-19 may develop conjunctivitis (3) but the viral positive rate of SARS$\mathrm{CoV}-2$ in tears and conjunctiva is reported to be very low (0 to $28 \%$ ) (4-11). Whether the ocular route plays a role in SARS-CoV-2 transmission remains unclear at present. Meanwhile, the viral load is reported to be much higher in the early phase of the disease (12), and therefore it is worth investigating whether the detection rate of SARS-CoV-2 on ocular surfaces is higher in the early stage of the disease.

Herein, we conducted a study to evaluate the viral positive rate on the ocular surfaces of patients with confirmed COVID-19 in different phases. According to the hospital procedures, the patients were classified into newly admitted patients and patients who had been hospitalized for more than 2 weeks. This study covered the broadest conjunctival sampling time between 1 day and 50 days from the illness onset. We present the following article in accordance with the MDAR reporting checklist (available at http://dx.doi.org/10.21037/atm-20-6026).

\section{Methods}

\section{Study population}

In this study, we recruited 59 COVID-19 patients who were hospitalized in the Shanghai Public Health Clinical Center (SPHCC), China between March 3, 2020, and March 21, 2020. All the diagnosis was confirmed according to the Chinese national guidelines for COVID-19. The mild type was defined as having slight clinical symptoms without pneumonia on radiography. The common type was defined as presenting with fever and/or respiratory symptoms plus pneumonia on radiography. Laboratory confirmations were achieved by the Chinese Center for Disease Control and Prevention (China CDC).

In these patients, 32 were newly admitted with hospitalization time from 1 to 2 days (mean \pm SD: $1.1 \pm 0.3$ days), while 27 were hospitalized for 17 to 41 days (mean \pm SD: $27.4 \pm 6.1$ days). All patients were Chinese. In newly admitted patients, 29 were imported cases from Great Britain [7], Italy [6], Iran [4], Spain [4], America [4], France [3], and Switzerland [1] (Table 1).

The study was conducted in accordance with the Declaration of Helsinki (as revised in 2013). The study was approved by the Ethics Committee of SPHCC (yj-2020-s047-01) and Eye and ENT Hospital of Fudan University (No. 2020020) and informed consent was taken from all the patients in advance for collecting their conjunctival swab samples.

\section{Main outcome measures}

A detailed and accurate epidemiological investigation was conducted by the CDC for each patient, including demographic characteristics, activity patterns, and clinical characteristics such as ocular symptoms and onset symptoms, etc. The temperature, laboratory findings, chest computed tomography (CT) scans, serum antibody, and treatment programs were obtained from the patients' electrical medical records.

Conjunctival swabs were taken from all enrolled patients 
Table 1 Baseline characteristics of patients with COVID-19

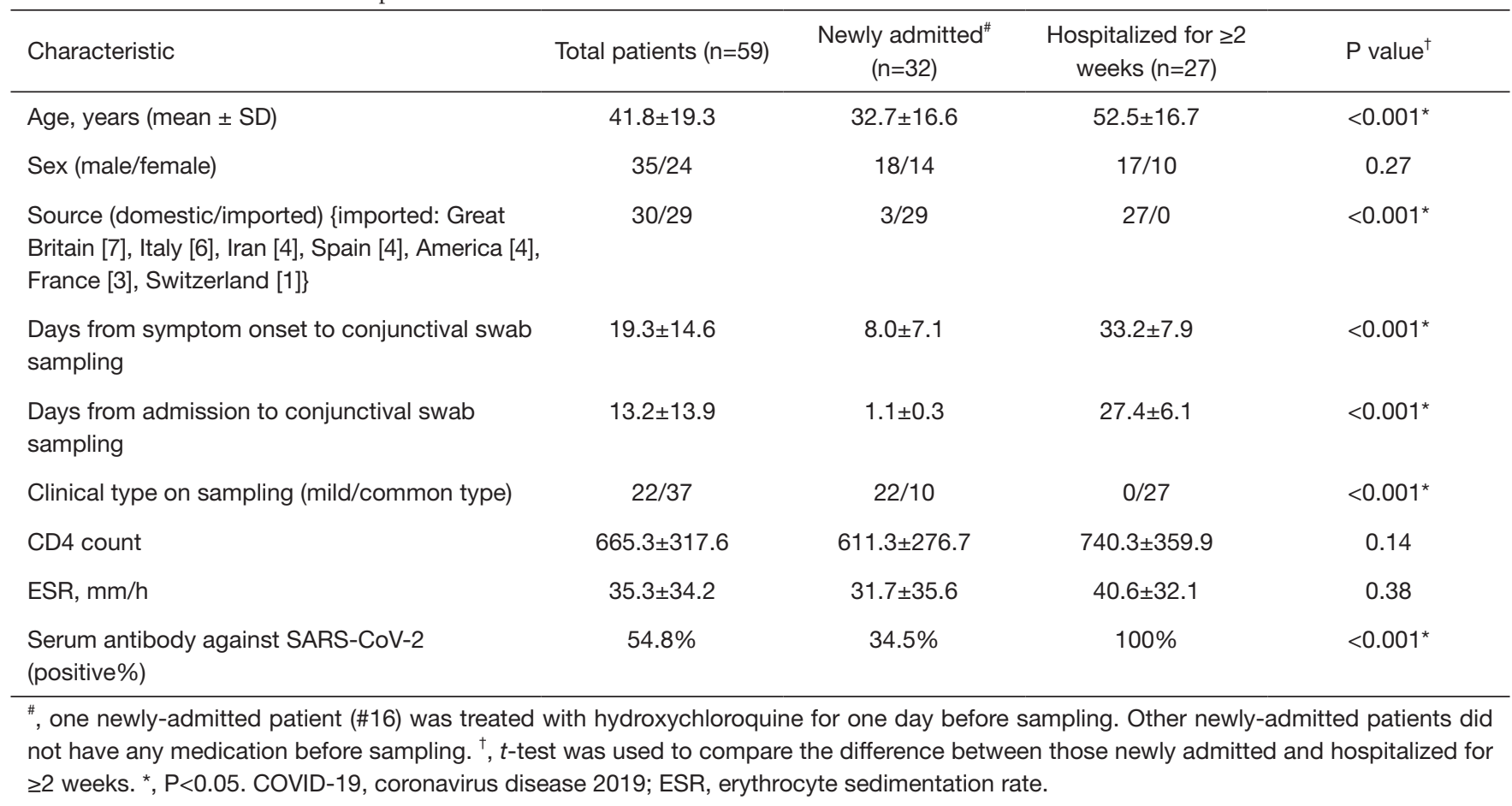

A

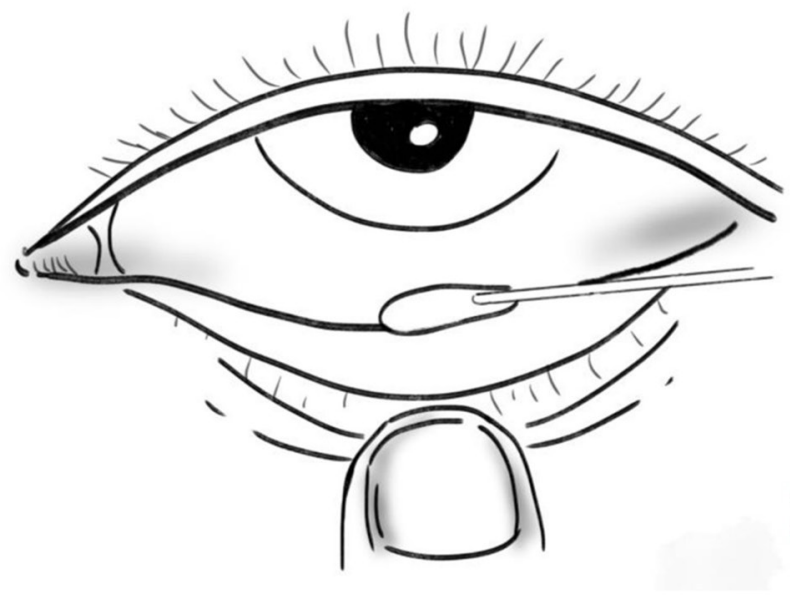

B

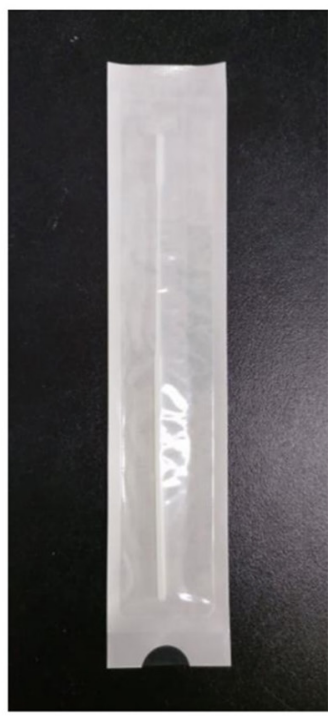

Figure 1 The swab. (A) Specimen collection location of the conjunctival swab; (B) Swab used in the present study. The figure was illustrated by the co-author Weiming Yang.

to collect tears, conjunctival secretion, and conjunctival cells. Specifically, we everted the inferior eyelid and rotated the sterile synthetic fiber swabs to softly wipe the conjunctiva of the lower eyelid fornix (Figure 1). Samples from each eye were taken and analyzed separately. Patients with positive results in conjunctival swabs were sampled again 1 week later, and the ocular examination was conducted every day until their discharge. Caution was 


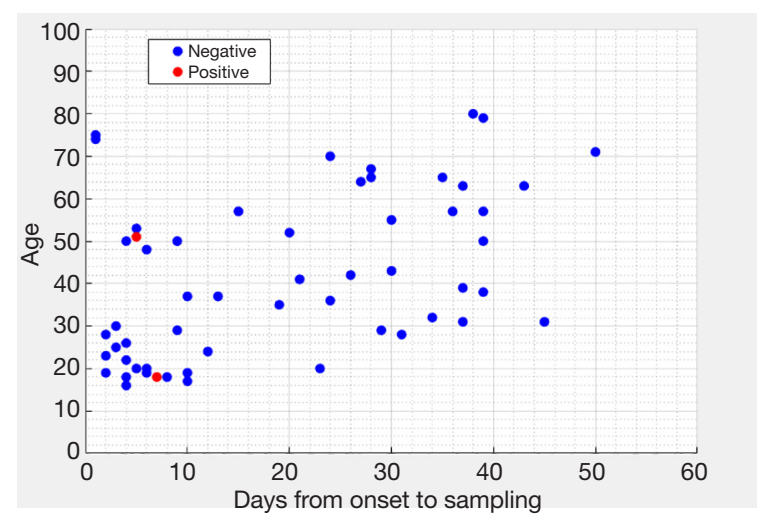

Figure 2 Patient characteristics, sampling time and results of conjunctival swabs.

taken to prevent contamination of samples. All procedures were performed by the same ophthalmologist at SPHCC, who specialized in infectious eye diseases and was familiar with the related sampling procedure. Meanwhile, the oropharyngeal swabs were routinely taken for every patient.

We extracted RNA from the swabs and conducted realtime reverse transcription polymerase chain reaction (RTPCR) for SARS-CoV-2 as previously described (13) by using reagents provided by Da An Gene Co., Ltd. (Sun YatSen University, http://en.daangene.com). All of the samples were tested in SPHCC's P3 laboratory by technicians with extensive experience in viral RNA testing.

\section{Statistical analysis}

Data were analyzed using SPSS version 22.0 for Windows (SPSS Inc., Chicago, IL, USA). Continuous and categorical variables were summarized by mean \pm standard deviation (SD) and frequencies respectively. The two-sample $t$-test and adjusted $t$-test were used to compare the means between groups for continuous variables. The chi-square tests were used to test the difference in frequency between groups for categorical variables. A two-tailed $\mathrm{P}$ value of $<0.05$ was considered statistically significant.

\section{Results}

The baseline characteristics are summarized in Table 1. The mean age of 59 patients was $41.8 \pm 19.3$ years. There were 35 males and 24 females. The conjunctival swabs tested for SARS-CoV-2 were positive in the 2 eyes of 2 newly admitted patients (\#44 and \#52), while none of the longer- stay patients had positive results (Figure 2). The mean time from symptom onset to conjunctival swab sampling of positive cases was 6.0 days ( $\mathrm{SE}=1.0$ ), which was significantly ( $\mathrm{P}$ value of a $t$-test $<0.001$ ) less than that of negative cases (mean $=19.7 ; \mathrm{SE}=2.0$ ). Details about these 2 positive patients are listed as follows.

Patient \#44 was a male, 51-year old, common type patient, and was admitted after he arrived in Shanghai from Spain. He had a cough and expectoration, but no fever, for 4 days before admission. The chest $\mathrm{CT}$ showed viral pneumonia. The oropharyngeal, right eye, and left eye's conjunctival swabs tested positive, negative, and weakly positive, respectively, for SARS-CoV-2 on the second day of admission (5 days after symptom onset). Both of his eyes were mildly congested. There was a pterygium in his right eye and a pinguecula in his left eye (Figure $3 A, B$ ). His body temperature at sampling was $36.4{ }^{\circ} \mathrm{C}$. The conjunctival swabs samples from his eyes turned negative 1 week later (12 days after symptom onset). During his whole 14-day hospital stay, no conjunctivitis symptoms were found.

Patient \#52 was a male, 18-year old, mild type patient, and was admitted after he arrived in Shanghai from Great Britain. He had a fever 6 days before admission, which was relieved without medication. He had a headache, but no fever or respiratory symptoms at admission. The chest $\mathrm{CT}$ was normal. Both the oropharyngeal and right eye conjunctival swabs tested positive for SARS-CoV-2 on the second day of admission (7 days after symptom onset), but there were no ocular symptoms or signs in either eye (Figure $3 C, D$ ). His body temperature at sampling was $36.5^{\circ} \mathrm{C}$. The conjunctival swab samples from his eyes turned negative 1 week later (14 days after symptom onset). During his whole 17-day hospital stay, no ocular symptoms were found.

\section{Discussion}

There is a high risk of SARS-CoV-2 transmission for healthcare workers, especially when they have direct contact with patients' mucosa or body fluids during diagnostic and therapeutic procedures. Therefore, there is an urgent need to evaluate the risk of infection during the exposure and to address the pattern of SARS-CoV-2's presence in the ocular surface.

In the current study, we investigated the impact of the sampling time on the detection rate for the first time, and found that the prevalence within 2 weeks of onset was as high as $7.1 \%(2 / 27)$. Both positive cases were newly 

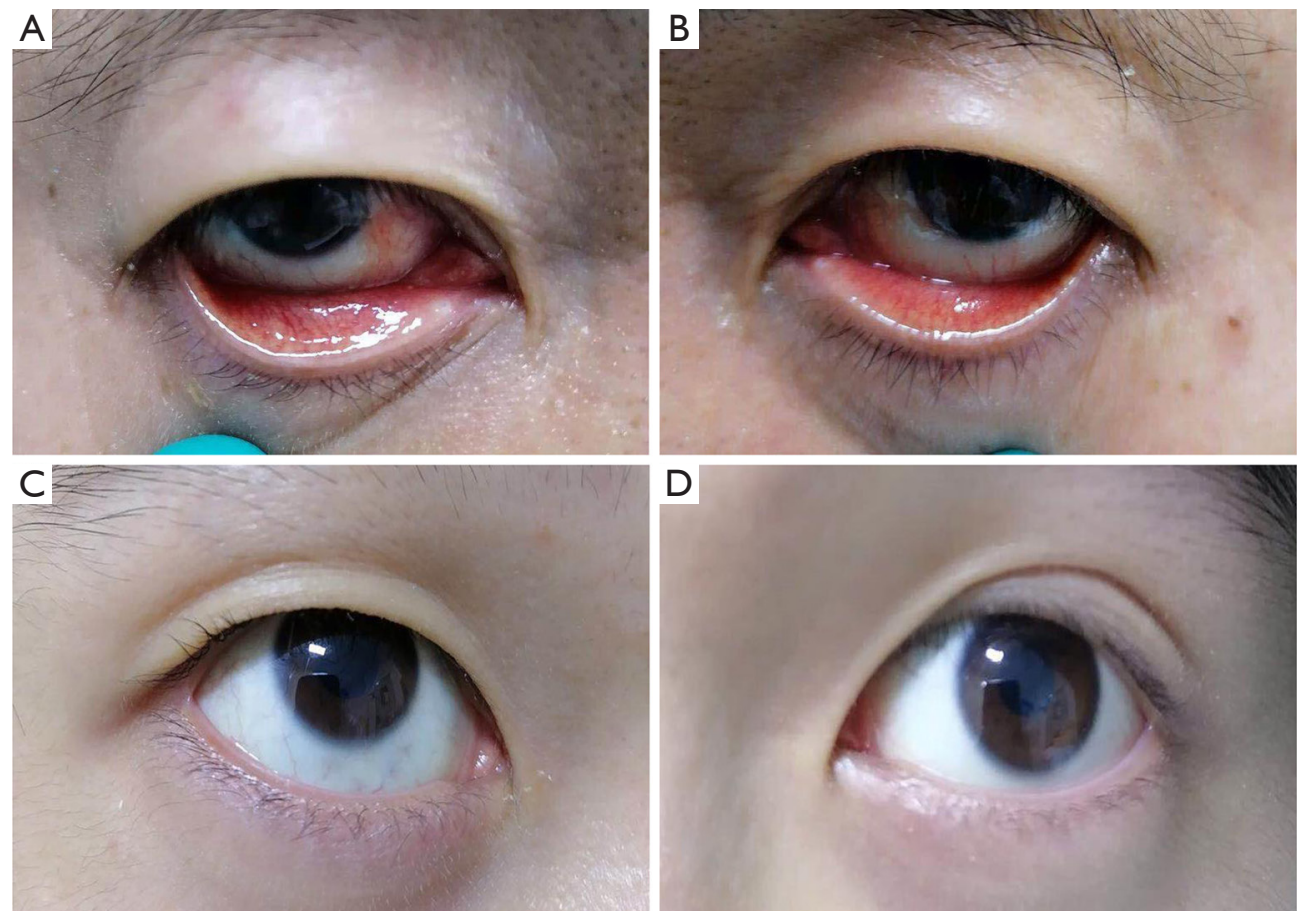

Figure 3 The clinical photographs of the positive patients. (A,B) (A: right eye; B: left eye): both eyes of patient \#44 were mildly congested. There was a pterygium in his right eye and a pinguecula in his left eye. The conjunctival swab sample from his left eye was positive at the first sampling, but was negative 1 week later; (C,D) (C: right eye; D: left eye): no ocular symptom was detected in either eye of the patient \#52, and the conjunctival swab sample from his right eye was positive at the first sampling, but was negative 1 week later.

admitted and had a shorter mean time from onset to sampling than that of negative cases. For both cases, the first tests of conjunctival swab samples were positive within 1 week of onset but the repeated tests after 1 week were negative. The finding indicates that the SARS-CoV-2 RNA is more likely to be detected on ocular surfaces for a short period of the disease duration. Several clinical studies $(4,7)$ and case reports $(14,15)$ are in line with our results. In these studies, the positive RT-PCR results of conjunctival swabs were also firstly detected within 2 weeks of symptom onset, and the positive case in the study of Zhang et al. (7) had a negative result when tested repeatedly on day 10 . Similarly, in the rhesus macaques inoculated with the Middle East respiratory syndrome coronavirus (MERS-CoV), a MERS viral load could only be detected at day 3 post-inoculation but not at day 6 in the conjunctiva (16). Wölfel et al. (12) reported that the peak SARS-CoV-2 RNA concentration in throat swab was reached before day 5 and was more than 1,000 times higher. Our results support it to some extent while suggesting that in the early stages of the disease when viral loads are high, it could be easier to detect the virus on ocular surfaces and that there is a higher risk of ocular transmission. Admittedly, there is still transmission risk of SARS-CoV-2 after 2 weeks and a case was reported with virus RNA detected 27 days after initial symptoms onset (17).

The positive rate of SARS-CoV-2 in ocular surfaces in the present study as well as others in the literature (4-11) is relatively low (we summarized studies regarding SARSCoV-2 in ocular surfaces in Table 2). Seah et al. reported neither viral culture nor RT-PCR detected the virus in 64 tear samples for 17 COVID-19 patients (8). Compared with their study, we used conjunctival swabs to collect not only tears but also conjunctival secretion and conjunctival cells, and detected 2 patients with positive RT-PCR results. However, the actual positive rate could be higher than that reported. Firstly, a certain false-negative rate in the viral RNA test was inevitable due to improper collection of clinical specimens or poor handling of specimens after collection (18). Secondly, recall bias could have affected the accuracy of symptom-onset dates reported by cases here, and the imported cases with reported onset after inbound 


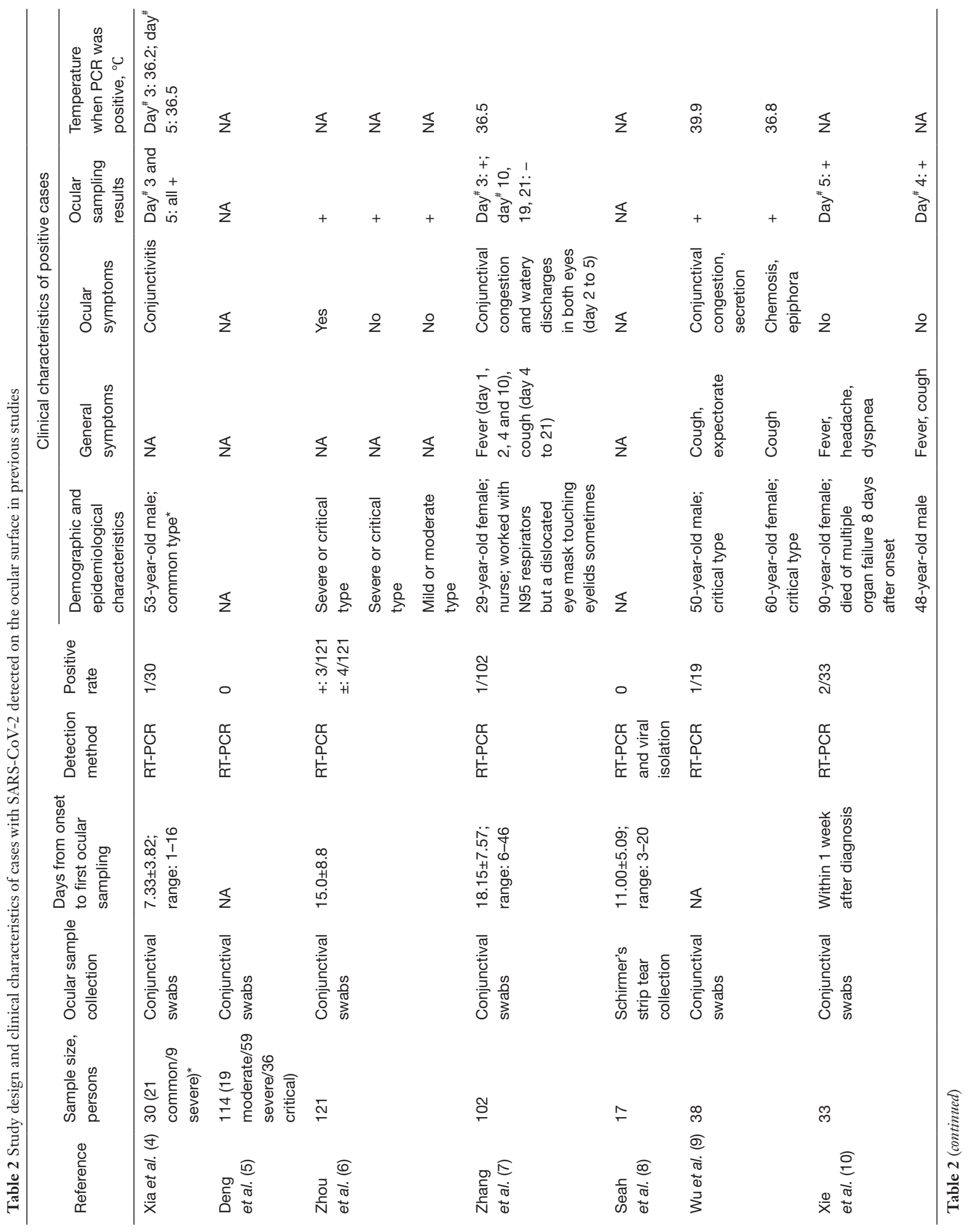




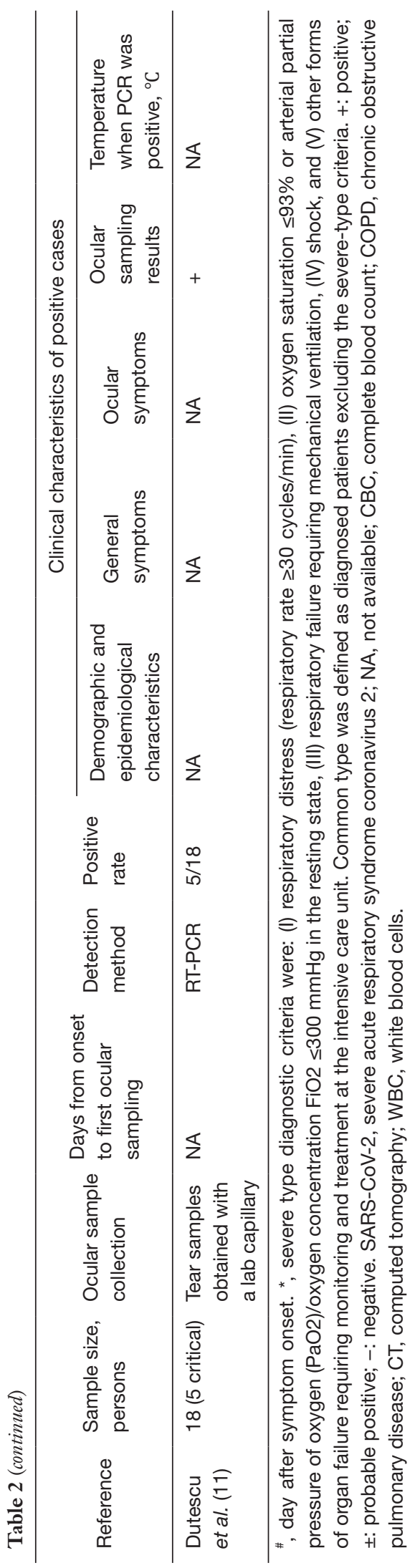

may have had initial symptoms even earlier. Therefore, the actual viral positive rate in ocular surface within 2 weeks of onset may have been underestimated.

Interestingly, some cases that tested positive for SARS$\mathrm{CoV}-2$ in the ocular surface had ocular signs or symptoms while others did not. In this study, both positive cases had no apparent conjunctivitis symptoms. According to the reported literature, 6 patients that tested positive for SARS-CoV-2 presented with conjunctivitis $(4,7,9,15)$ or keratoconjunctivitis (14), and 2 of them had it as the initial symptom, but another 4 patients $(6,10)$ were asymptomatic, which is in line with our result. The possible reason for the difference in ocular manifestations might be that conjunctival or corneal epitheliums are not highly susceptible to SARS-CoV-2 or that the viral load on the ocular surface does not reach the pathogenic dose. Considering the co-expression of SARS-CoV-2 entry receptor ACE2 and viral entry-associated protease TMPRSS2 in superficial conjunctival cells (19), the ocular surface still might represent niches for virus replication. The relationship between viral loads and ocular symptoms currently remains unanswered.

Moreover, the detection of SARS-CoV-2 RNA in conjunctival swabs suggests that the ocular surface may also serve as either a portal of viral entry or as a reservoir secreting infective agents. SARS-CoV-2 is highly contagious and during close contact with patients, the conjunctiva is easily exposed to infectious droplets and fomites. Anatomically, the ocular surface is linked with the upper respiratory tract, primarily by the nasolacrimal system (20). Deng et al. (21) successfully inoculated the SARS-CoV-2 virus to rhesus macaques via the conjunctival route, which also suggested that conjunctiva was a portal for viral transmission. Additionally, some respiratory viruses, such as the respiratory syncytial virus (22), were isolated in tears, and therefore, the eye could also serve as sources of contamination. Further mechanistic studies on the role of the ocular surface in SARS-CoV-2 transmission are warranted.

There are several strengths and limitations to this study. A salient feature of our study is that the sampling time of conjunctival swabs covered between 1 day and 50 days from the illness onset, which is the broadest sampling time yet. Secondly, the detailed and accurate epidemiological investigation by CDC ensures the reliability of the data of this study, including the time of onset. There is one thing to note that, in order to provide patient comfort and protection, repeat sampling of conjunctival tissues was 
avoided for cases that were initially tested negative.

In conclusion, this study provides a new insight that SARS-CoV-2 on the ocular surface may be more likely to be detectable in a short period from disease onset. The risk of ocular transmission could be higher in the early phase and is likely to be underestimated yet. Therefore, eye protection (goggles or face shield), hand hygiene, and other personal protection procedures are recommended to healthcare workers who are in direct contact with patients' ocular surfaces or at the risk of splash exposure of eye secretions. It is advisable to avoid nonemergent ocular surgeries and nonessential examinations. For a COVID-19 patient, avoiding touching one's eyes and frequent hand washing is essential to help stop the spread of the virus.

\section{Acknowledgments}

The authors would like to thank Prof. Bo Fu (School of Data Science, Fudan University) for providing valuable statistical consultations. We express our sincere wishes and greatest respect to the front-line workers, who are fighting against COVID-19. We thank William Pelham COON, MS, MBA, PhD, retired professor at Washington University in St. Louis for English language editing.

Funding: This study was supported by the Second Batch of Emergency Project of the Shanghai Science and Technology Committee (20411950200) and the Special Emergency Project for the Prevention and Treatment of COVID-19 with Traditional Chinese Medicine in Shanghai (2020NCP001).

\section{Footnote}

Reporting Checklist: The authors have completed the MDAR reporting checklist. Available at http://dx.doi.org/10.21037/ atm-20-6026

Data Sharing Statement: Available at http://dx.doi. org/10.21037/atm-20-6026

Conflicts of Interest: All authors have completed the ICMJE uniform disclosure form (available at http://dx.doi. org/10.21037/atm-20-6026). The authors have no conflicts of interest to declare.

Ethical Statement: The authors are accountable for all aspects of the work in ensuring that questions related to the accuracy or integrity of any part of the work are appropriately investigated and resolved. The study was conducted in accordance with the Declaration of Helsinki (as revised in 2013). The study was approved by the Ethics Committee of SPHCC (yj-2020-s047-01) and Eye and ENT Hospital of Fudan University (No. 2020020) and informed consent was taken from all the patients in advance for collecting their conjunctival swab samples. Informed consent was obtained from all participants.

Open Access Statement: This is an Open Access article distributed in accordance with the Creative Commons Attribution-NonCommercial-NoDerivs 4.0 International License (CC BY-NC-ND 4.0), which permits the noncommercial replication and distribution of the article with the strict proviso that no changes or edits are made and the original work is properly cited (including links to both the formal publication through the relevant DOI and the license). See: https://creativecommons.org/licenses/by-nc-nd/4.0/.

\section{References}

1. Li Q, Guan X, Wu P, et al. Early Transmission Dynamics in Wuhan, China, of Novel Coronavirus-Infected Pneumonia. N Engl J Med 2020;382:1199-207.

2. Lu CW, Liu X, Jia Z. 2019-nCoV transmission through the ocular surface must not be ignored. Lancet 2020;395:e39.

3. Guan WJ, Ni ZY, Hu Y, et al. Clinical Characteristics of Coronavirus Disease 2019 in China. N Engl J Med 2020;382:1708-20.

4. Xia J, Tong J, Liu M, et al. Evaluation of coronavirus in tears and conjunctival secretions of patients with SARSCoV-2 infection. J Med Virol 2020;92:589-94.

5. Deng C, Yang Y, Chen H, et al. Low risk of SARS-CoV-2 transmission through the ocular surface. Acta Ophthalmol 2020;98:e926-7.

6. Zhou Y, Duan C, Zeng Y, et al. Ocular Findings and Proportion with Conjunctival SARS-COV-2 in COVID-19 Patients. Ophthalmology 2020;127:982-3.

7. Zhang X, Chen X, Chen L, et al. The evidence of SARS-CoV-2 infection on ocular surface. Ocul Surf 2020;18:360-2.

8. Seah IYJ, Anderson DE, Zheng Kang AE, et al. Assessing Viral Shedding and Infectivity of Tears in Coronavirus Disease 2019 (COVID-19) Patients. Ophthalmology 2020;127:977-9.

9. Wu P, Duan F, Luo C, et al. Characteristics of Ocular Findings of Patients With Coronavirus Disease 
2019 (COVID-19) in Hubei Province, China. JAMA Ophthalmol 2020;138:575-8.

10. Xie HT, Jiang SY, Xu KK, et al. SARS-CoV-2 in the ocular surface of COVID-19 patients. Eye and Vision (London, England) 2020;7:23.

11. Dutescu RM, Banasik P, Schildgen O, et al. Detection of coronavirus in tear samples of hospitalized patients with confirmed SARS-CoV-2 from oropharyngeal swabs. Cornea 2020;10.1097/ICO.0000000000002562.

12. Wölfel R, Corman VM, Guggemos W, et al. Virological assessment of hospitalized patients with COVID-2019. Nature 2020;581:465-9.

13. Huang C, Wang Y, Li X, et al. Clinical features of patients infected with 2019 novel coronavirus in Wuhan, China. Lancet 2020;395:497-506.

14. Cheema M, Aghazadeh H, Nazarali S, et al. Keratoconjunctivitis as the initial medical presentation of the novel coronavirus disease 2019 (COVID-19). Can J Ophthalmol 2020;55:e125-9.

15. Chen L, Liu M, Zhang Z, et al. Ocular manifestations of a hospitalised patient with confirmed 2019 novel coronavirus disease. Br J Ophthalmol 2020;104:748-51.

16. de Wit E, Rasmussen AL, Falzarano D, et al. Middle

Cite this article as: Li M, Yang Y, He T, Wei R, Shen Y, Qi T, Han T, Song Z, Zhu Z, Ma X, Zhao J, Lin Y, Shen Y, Yang W, Zhao K, Lu H, Zhou X. Detection of SARS-CoV-2 in the ocular surface in different phases of COVID-19 patients in Shanghai, China. Ann Transl Med 2021;9(2):100. doi: 10.21037/atm-20-6026
East respiratory syndrome coronavirus (MERS-CoV) causes transient lower respiratory tract infection in rhesus macaques. Proc Natl Acad Sci 2013;110:16598-603.

17. Colavita F, Lapa D, Carletti F, et al. SARS-CoV-2 Isolation From Ocular Secretions of a Patient With COVID-19 in Italy With Prolonged Viral RNA Detection. Ann Intern Med 2020;173:242-3.

18. Lin C, Ye R, Xia YL. A meta-analysis to evaluate the effectiveness of real-time PCR for diagnosing novel coronavirus infections. Genet Mol Res 2015;14:15634-41.

19. Sungnak W, Huang N, Bécavin C, et al. SARS-CoV-2 entry factors are highly expressed in nasal epithelial cells together with innate immune genes. Nat Med 2020;26:681-7.

20. Belser JA, Rota PA, Tumpey TM. Ocular Tropism of Respiratory Viruses. Microbiol Mol Biol Rev 2013;77:144-56.

21. Deng $W$, Bao L, Gao H, et al. Ocular conjunctival inoculation of SARS-CoV-2 can cause mild COVID-19 in rhesus macaques. Nat Commun 2020;11:4400.

22. Fujishima H, Okamoto Y, Saito I, et al. Respiratory syncytial virus and allergic conjunctivitis. J Allergy Clin Immunol 1995;95:663-7. 